\title{
Prevalencia de helmintos intestinales en gatos domésticos del departamento del Quindío, Colombia
}

\author{
Diana Marcela Echeverry, María Isabel Giraldo, Jhon Carlos Castaño \\ Grupo de Inmunología Molecular, Centro de Investigaciones Biomédicas, Facultad Ciencias de la Salud, \\ Universidad del Quindío, Armenia, Colombia
}

Introducción. Las enfermedades producidas por helmintos están ampliamente distribuidas en el mundo y muchas de ellas se consideran zoonosis. Los animales de compañía cumplen un papel trascendental en la transmisión a los humanos.

Objetivo. Determinar la prevalencia de helmintos intestinales en gatos del departamento del Quindío. Materiales y métodos. Se estudiaron 121 gatos domésticos del departamento del Quindío, de los cuales se registraron los datos de sexo, edad y condición corporal. Se recolectaron heces y se procesaron mediante las técnicas de Ritchie modificada y de Kato-Katz.

Resultados. De los 121 gatos, 42,14 \% (IC I5\%: 33,35-50,94) y 45,45 \% (IC $\left._{95 \%}: 36,58-54,32\right)$ resultaron parasitados con alguna especie de helminto adulto según la presencia de huevos en sus heces, mediante las técnicas de Ritchie y de Kato-Katz, respectivamente. Toxocara cati fue el parásito más prevalente (Ritchie: 37,2 \%; Kato-Katz: 43 \%), seguido por Ancylostoma spp. (Ritchie: 7,43 \%; KatoKatz: $5,78 \%$ y Aelurostrongylus abstrusus (Ritchie: 0,82 \%).

Sesenta y cinco $(53,71 \%)$ gatos eran hembras y $56(46,28 \%)$ eran machos; la prevalencia de infección fue similar en ambos sexos. Los felinos mayores de 4 años de edad presentaron mayor prevalencia de parásitos (81,8 \%), seguidos por los de 1 a 4 años (48,8\%) y, por último, por los menores de un año (28,6 \%). Se encontró una buena condición corporal en 77,68 \% y este grupo presentó menor frecuencia de helmintos intestinales.

Conclusión. La prevalencia de helmintos intestinales en gatos domésticos del departamento del Quindío fue de 43,8 \%, lo que hace necesario establecer programas de vigilancia y prevención en la población humana y felina.

Palabras clave: helmintos, helmintiasis/epidemiología, enfermedades de los gatos, Ancylostoma, Toxocara. doi: http://dx.doi.org/10.7705/biomedica.v32i3.439

\section{Prevalence of intestinal helminths in cats in Quindío, Colombia}

Introduction. Diseases caused by helminths are widely distributed in the world and many of them are considered zoonoses in which pets play a major role in transmission to humans.

Objective. The prevalence of intestinal helminths was determined in cats in Quindío Province.

Materials and methods. One hundred twenty-one cats were characterized --data recorded included sex, age and body condition. Fecal samples were collected and processed using the modified Ritchie and modified Kato-Katz techniques to determine the presence of intestinal helminths.

Results. Of the 121 cats, $42.1 \%$, (95\% Cl: 33.4-50.9) and $45.5 \%(95 \% \mathrm{Cl}: 36.6-54.3)$ were parasitized with at least one adult helminth species as evidenced by the presence of eggs in their fecal samples. Toxocara cati was the most prevalent parasite (Ritchie: $37.2 \%$, Kato-Katz: 43\%), followed by Ancylostoma spp. (Ritchie: 7.4\%, Kato-Katz: 5.8\%) and Aelurostrongylus abstrusus (Ritchie: 0.82\%). Sixty-five cats (53.7\%) were females and 56 (46.3\%) males; the prevalence of infection was similar in both sexes. Cats older than 4 years had the highest prevalence $(81.8 \%)$ followed by those aged 1 to 4 years (48.8\%) and by those under 1 year (28.6\%). The majority of cats, $77.7 \%$, were found to be in good body condition and this group had the lowest frequency of intestinal helminths with both techniques.

Conclusion. The prevalence of intestinal helminths in domestic cats in Quindío was $43.8 \%$; it is necessary to establish surveillance and prevention programs in the human and feline populations.

Key words: Helminths, helminthiasis/epidemiology, cat diseases, Ancylostoma, Toxocara.

doi: http://dx.doi.org/10.7705/biomedica.v32i3.439

\footnotetext{
Contribución de los autores:

Diana Marcela Echeverry: toma, procesamiento de muestras y participación en La elaboración de la primera versión del manuscrito.

María Isabel Giraldo: procesamiento de muestras y revisión del manuscrito.

Jhon Carlos Castaño: revisión y procesamiento de datos y elaboración de manuscrito.
} 
Las enfermedades parasitarias se encuentran ampliamente distribuidas en el mundo y muchas se constituyen en zoonosis, entidades definidas como infecciones e infestaciones que, en condiciones naturales, se transmiten de los animales vertebrados al hombre o inversamente. Los animales de compañía juegan un papel trascendental en la sociedad ya que benefician el desarrollo social, emocional y, en general, el bienestar de sus propietarios, aunque, a pesar de estos beneficios, pueden también constituirse como un riesgo potencial para la salud de quienes conviven con ellos. Los propietarios de los animales desconocen los peligros que sus mascotas parasitadas representan para su salud y la de su familia (1).

La población de gatos domésticos (Felis catus domesticus) utilizados como animales de compañía, se ha incrementado al igual que la necesidad de lograr su bienestar (2). La mayoría de gatos domésticossontratadoscomomiembros delafamilia, permitiéndoles compartir la misma habitación y hasta el mismo espacio para dormir, sin considerar el posible riesgo de transmisión de enfermedades zoonóticas. Esta especie animal puede ser portadora de enfermedades virales, bacterianas y parasitarias que afectan principalmente a los niños, ya que este grupo se expone a un mayor contacto con los excrementos de los gatos, debido a la contaminación de parques, campos de juego, calles y jardines con las heces de estos animales (3).

Un aspecto muy importante que se debe tener en cuenta con respecto a los helmintos gastrointestinales de gatos, es su carácter zoonótico. Es bien conocido que la larva migrans cutánea es, principalmente, producida por Ancylostoma braziliense (4), el cual es uno de los Ancylostoma más comunes en gatos. Por otra parte, a pesar de que Toxocara canis es el áscaris que más comúnmente causa larva migrans visceral, Toxocara cati es potencialmente capaz de producir esta condición. De hecho, recientemente después de comprobarse a T. cati como el agente causal en algunos casos humanos de larva migrans visceral y larva migrans ocular (5), se ha empezado a reconocer a este nematodo como un agente zoonótico de gran importancia en salud pública.

Correspondencia:

Jhon Carlos Castaño, Facultad de Medicina, Universidad del Quindío, Carrera 15 calle 12 norte, Armenia, Colombia

Fax: (576) 7460129

jhoncarlos@uniquindio.edu.co

Recibido: 07/09/11; aceptado:27/04/12
En el caso de los gatos, existen factores propios de la especie que favorecen la transmisión de los helmintos intestinales, como su carácter "nómada" y el instinto cazador. Esta práctica "natural" en el gato y muchas veces hasta bien vista por sus dueños, lleva implícita una serie de riesgos y peligros potenciales para el mismo gato, para sus propietarios (zoonosis), para otros gatos de la zona y para la salud pública en general (1); estas incursiones callejeras comienzan alrededor de los seis a los ocho meses de vida, que es el momento en que se produce la pubertad del gato. Es de hacer notar que aunque el gato tenga cubierta su ración alimenticia en la casa que comparte con los humanos, no se inhibe totalmente la conducta predatoria, ya que sus mecanismos son independientes; en otras palabras, la saciedad no inhibe la conducta de cazador. El sistema gastrointestinal de los felinos está expuesto de manera constante a la colonización de varios agentes patógenos, entre los cuales los helmintos son los más comunes (6), siendo causa frecuente de enfermedad en gatos adultos y en cachorros (7).

En vista de la importancia que tienen los helmintos intestinales en gatos y que son reconocidos como agentes de gran importancia en la medicina veterinaria y en salud pública, por ser potencialmente zoonóticos y teniendo en cuenta que en el departamento del Quindío no existen reportes sobre la prevalencia de estos agentes patógenos, se determinó la prevalencia de parásitos intestinales en gatos domésticos de este departamento.

\section{Materiales y métodos}

Se llevó a cabo un estudio prospectivo de tipo descriptivo en la población felina con dueño que fue registrada en la jornada de vacunación antirrábica del 2008 en departamento del Quindío. Para determinar la prevalencia de helmintos intestinales, se tomó como universo de estudio 9.787 gatos, reportados por el Instituto Seccional de Salud del Quindío y la Secretaría de Salud de Armenia, en el perfil epidemiológico del Quindío del 2008. A partir de estos datos se hizo un muestreo aleatorio estratificado con un error del $5 \%$ y un nivel de confianza del $95 \%$, estudiando 121 gatos. A cada uno de los propietarios se les invitó a participar voluntariamente en el estudio, y una vez habían aceptado, se les explicó el procedimiento que se iba a seguir. Posteriormente, se hizo la encuesta donde se consignaron los datos del animal. 


\section{Toma y análisis de las muestras}

De cada gato seleccionado se obtuvo una muestra de heces, la cual se envasó en un recipiente limpio, de boca ancha y de cierre hermético, rotulado adecuadamente con los siguientes datos: nombre del animal, fecha de recepción de la muestra y dirección de domicilio. Posteriormente, las muestras se transportaron al Centro de Investigaciones Biomédicas de la Universidad del Quindío, donde se guardaron en neveras a $4{ }^{\circ} \mathrm{C}$ hasta ser procesadas utilizando las técnicas de Kato-Katz y la técnica de Ritchie modificada.

La identificación de los parásitos se basó en sus características morfológicas y en las mediciones biométricas de los huevos en el microscopio, además de ser comparados con fotografías estándar. Un animal fue considerado positivo cuando se observaba, al menos, un huevo de helminto en la muestra de heces.

\section{Análisis de la información}

Los datos de la encuesta y los resultados del examen coprológico se organizaron en una base de datos en el programa Excel ${ }^{\mathrm{TM}}$, versión 7 (Microsoft Office). Se calcularon las frecuencias y se obtuvo la prevalencia de parásitos por especie y huésped. Para la obtención de la prevalencia se utilizó la fórmula descrita por Thrusfield (8) y se calcularon los $\mathrm{IC}_{95 \%}$ de las prevalencias obtenidas. Luego se relacionaron las variables frente a los resultados obtenidos y se hizo un análisis de regresión logística con el programa Stagraphics Centurion $X^{\top \mathrm{M}}$.

\section{Resultados}

Se estudiaron 121 gatos del departamento del Quindío, sin distinción de edad ni sexo. El 42,14 \%
$\left(\mathrm{IC}_{95 \%}: 33,35-50,94\right)$ y $45,45 \%\left(\mathrm{IC}_{95 \%}: 36,58-54,32\right)$ resultaron parasitados con alguna especie de helminto adulto, según la presencia de huevos en sus heces, procesadas mediante la técnica de concentración de Ritchie y la técnica de KatoKatz.

En el cuadro 1 se presentan las características de los felinos con respecto al sexo y la presencia de parásitos. Se puede observar que 65 gatos $(53,71 \%)$ eran hembras y $56(46,28 \%)$ eran machos. El porcentaje de animales con helmintos fue mayor en hembras que en machos, conn ambas técnicas (Ritchie: 46,15\%; Kato-Katz: 47,69\%).

Con relación a los rangos de edad de los animales estudiados, aquellos cuya edad estaba entre 1 y 4 años presentaron una mayor prevalencia de parásitos, 46,34\%, con la técnica de Ritchie, mientras que los gatos mayores de 4 años de edad presentaron una mayor prevalencia de parásitos, $81,81 \%$ con la técnica de Kato-Katz; las menores prevalenciasseobservaronenlosanimalesmenores de 1 año: $28,57 \%$ y $21,42 \%$ con las técnicas de Ritchie y Kato-Katz, respectivamente. En cuanto a la condición corporal de los animales estudiados se encontró que el $77,68 \%$ presentaba una buena condición corporal, e igualmente, fueron los animales que presentaron menor frecuencia de helmintos intestinales diagnosticados con ambas técnicas (cuadro 1).

\section{Presencia de parásitos}

Las especies de helmintos encontradas en las heces de los felinos estudiados se presentan en la cuadro 2; $T$. cati fue el parásito más prevalente según las dos técnica utilizadas, Ritchie (37,19\%) y Kato-Katz (42,97\%), seguido por Ancylostoma

Cuadro 1. Distribución según algunas variables epidemiológicas de 121 felinos muestreados parasitológicamente en el departamento del Quindío

\begin{tabular}{|c|c|c|c|c|c|c|c|c|}
\hline \multirow{3}{*}{$\begin{array}{l}\text { Variable } \\
\text { Sexo }\end{array}$} & \multirow{3}{*}{ Total } & \multicolumn{7}{|c|}{ Número de animales con examen coprológico positivo para helmintos } \\
\hline & & \multicolumn{2}{|c|}{$\begin{array}{c}\text { Técnica de } \\
\text { concentración de Ritchie }\end{array}$} & \multicolumn{2}{|c|}{$\begin{array}{l}\text { Técnica de } \\
\text { Kato-Katz }\end{array}$} & \multicolumn{2}{|c|}{$\begin{array}{l}\text { Por cualquier } \\
\text { método }\end{array}$} & \multirow[t]{2}{*}{ OR $\left(\right.$ IC $\left._{95 \%}\right)$} \\
\hline & & $n$ & $(\%)$ & $\mathbf{n}$ & (\%) & $\mathbf{n}$ & (\%) & \\
\hline Hembras & 65 & 30 & $(46,1)$ & 31 & $(47,7)$ & 31 & $(47,7)$ & \\
\hline Machos & 56 & 21 & $(37,5)$ & 24 & $(42,8)$ & 24 & $(42,8)$ & $1,22(0,56-2,66)$ \\
\hline \multicolumn{9}{|l|}{ Edad } \\
\hline De 1 mes a 1 año & 28 & 8 & $(28,6)$ & 6 & $(21,4)$ & 8 & $(28,6)$ & Grupo de referencia \\
\hline De 1 año a 4 años & 82 & 38 & $(46,3)$ & 40 & $(48,8)$ & 40 & $(48,8)$ & $2,38(0,87-6,69)$ \\
\hline Mayor de 4 años & 11 & 5 & $(45,4)$ & 9 & $(81,8)$ & 9 & $(81,8)$ & $11,25(1,64-97,72)$ \\
\hline \multicolumn{9}{|l|}{ Condición corporal } \\
\hline Mala & 5 & 4 & $(80,0)$ & 3 & $(60,0)$ & 4 & $(80,0)$ & $5,64(0,56-137,85)$ \\
\hline Regular & 22 & 12 & $(54,5)$ & 14 & $(63,6)$ & 14 & $(63,6)$ & $2,47(0,86-7,20)$ \\
\hline Buena & 94 & 35 & $(37,2)$ & 39 & $(41,5)$ & 39 & $(41,5)$ & Grupo de referencia \\
\hline
\end{tabular}


spp. (Ritchie: 7,43 \%; Kato-Katz: 5,78 \%) y, finalmente, $A$. abstrusus (0,82 \%); este último se evidenció solamente mediante la técnica de concentración de Ritchie. Se observó biparasitismo en el $4,13 \%$ y $2,47 \%$ de las heces de los animales estudiados con las técnicas de Ritchie y Kato-Katz, respectivamente (cuadro 2, figuras 1-3).

Además, solo mediante la técnica de concentración de Ritchie se pudo observar la presencia de ooquistes de Toxoplasma gondii $(33,05 \%)$ y quistes de Giardia spp. (14,8\%) (no se presentan los datos).

\section{Factores asociados a la prevalencia}

En el cuadro 1 se resumen los resultados del análisis de asociación entre las variables epidemiológicas y los resultados de las prevalencias determinadas con las dos técnicas utilizadas en este estudio. La presencia de parásitos estuvo asociada a la condición corporal mala, con una alta significancia estadística para la técnica de Ritchie $(p=0,0005)$ y para la técnica de Kato-Katz $(p=0,0058)$. Los resultados con respecto a la edad fueron muy similares con ambas técnicas de diagnóstico. Mediante la técnica de Ritchie se encontraron 51 animales parasitados, 30 hembras y 21 machos, mientras con la técnica de Kato-Katz se encontraron 55, 31 hembras y 24 machos. Al comparar los porcentajes de animales parasitados por sexo, no se obtuvieron diferencias con significancia estadística.

Cuando se relacionaron los animales parasitados con la condición corporal y la convivencia con otros animales, no se encontraron diferencias estadísticamente significativas ( $p>0,05)$.

Al comparar los porcentajes de los felinos que presentaron parásitos adultos con los que presen-

Cuadro 2. Asociaciones parasitarias de los felinos estudiados en el departamento del Quindío con números positivos y sus respectivas prevalencias, utilizando la técnica de Ritchie y la de Kato-Katz

\begin{tabular}{lrrrr}
\hline Parásito & \multicolumn{3}{c}{$\begin{array}{c}\text { Animales con examen coprológico } \\
\text { positivo para helmintos }\end{array}$} \\
\cline { 2 - 5 } & $\begin{array}{c}\text { Técnica de } \\
\text { concentración } \\
\text { de Ritchie }\end{array}$ & $\begin{array}{c}\text { Técnica de } \\
\text { Kato-Katz }\end{array}$ \\
\hline Monoparasitismo & $\mathbf{n}$ & $\%$ & $\mathbf{n}$ & $\%$ \\
Toxocara cati & 45 & 37,19 & 52 & 42,97 \\
Ancylostoma spp. & 9 & 7,43 & 7 & 5,78 \\
$\begin{array}{l}\text { Aelurostrongylus abstrusus } \\
\text { Biparasitismo }\end{array}$ & 1 & 0,82 & 0 & 0 \\
Toxocara cati y & & & & \\
Ancylostoma spp. & 5 & 4,13 & 3 & 2,47 \\
\hline
\end{tabular}

taban diarrea, se halló una razón de probabilidad de $4,2$ ( $p<0,05)$, por lo que la probabilidad de encontrar que un gato con diarrea haya eliminado parásitos adultos es cuatro veces más alta comparada con la de un gato sin diarrea.

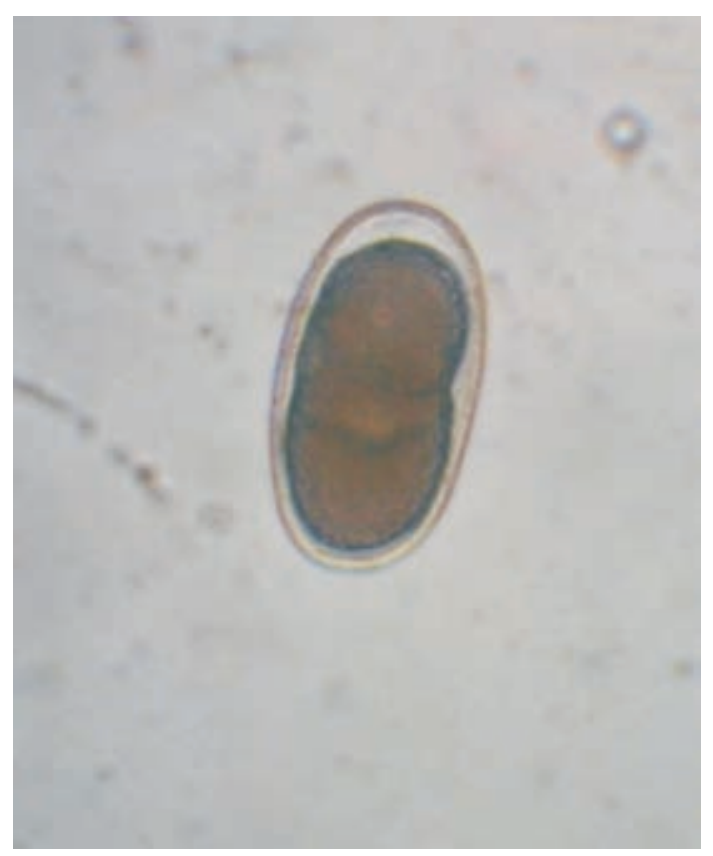

Figura 1. Huevo de Ancylostoma spp. Microscopio invertido Olympus CK2 ${ }^{\mathrm{TM}}$, 400X.

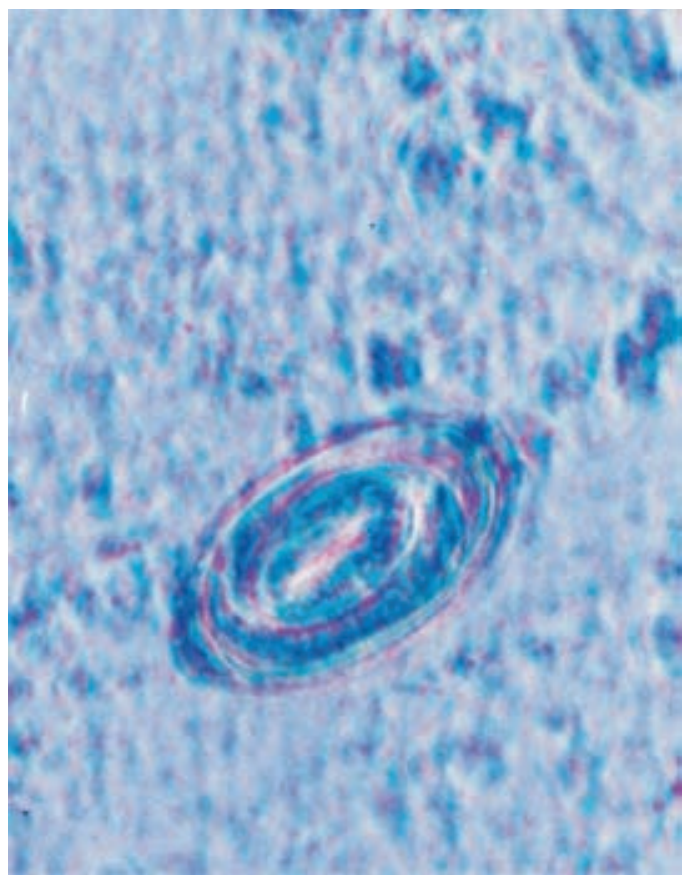

Figura 2. Huevo de Ancylostoma spp. con embrión. Microscopio invertido Olympus CK2 ${ }^{\mathrm{TM}}$, 400X. 


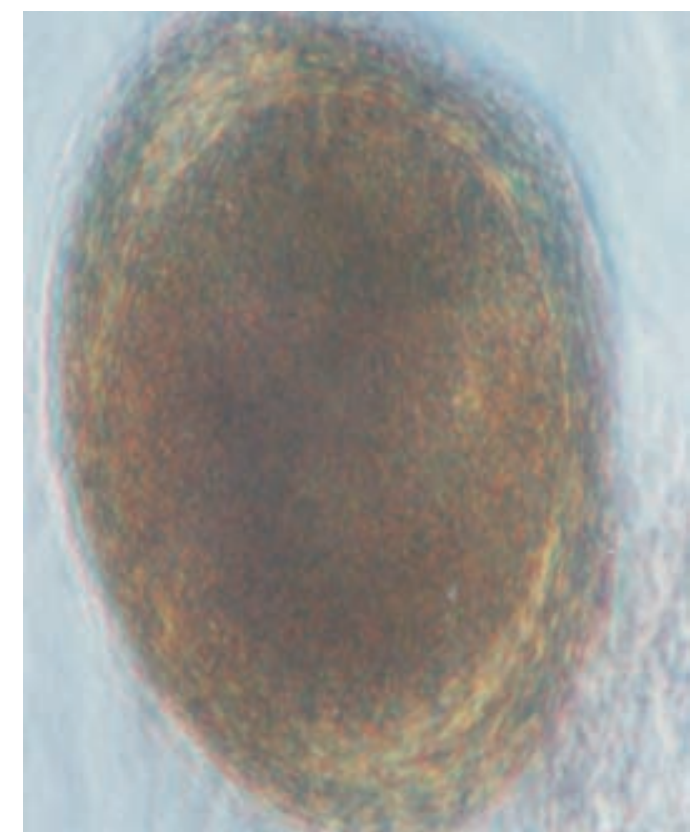

Figura 3. Huevo de Toxocara cati. Microscopio invertido Olympus CK2 ${ }^{\mathrm{TM}}$, 400X.

\section{Discusión}

En todo el mundo se han reportado helmintos intestinales en gatos, con prevalencias que varían entre $24 \%$ y $90 \%$. Estas cifras de prevalencia se han determinado por medio del análisis de materia fecal y en la inspección post mórtem (9-12).

En el departamento del Quindío, Giraldo, et al., en el 2003, reportaron prevalencia de parásitos intestinales en 19 gatos de Armenia, con los siguientes valores: Ancylostoma spp., 5,6 \%; T. gondii, 22,2 \%; Strongyloides spp., 5,6 \%; T. cati, 1,6\%, y Cystoisospora spp., $18 \%$ (13).

En el presente estudio se encontraron especies similares a las reportadas para Armenia por estos investigadores. Al analizar la prevalencia por especie de helmintos intestinales, los resultados encontrados en este estudio para $T$. cati son superiores a los reportados previamente en Armenia (14). Esto podría atribuirse al número de muestras y a las variaciones ambientales, principalmente de temperatura y humedad, dado que el presente estudio se llevó a cabo en todo el departamento del Quindío. Se ha reportado que las parasitosis son más frecuentes en las áreas tropicales y subtropicales (15), porque sus condiciones propician el desarrollo y la persistencia parasitaria, debido a que los helmintos necesitan ambientes cálidos y húmedos. De igual manera, estas condiciones climáticas son propicias para la supervivencia de ratones, cucarachas, lagartijas etc., que actúan como huéspedes intermediarios en algunas parasitosis, lo cual incrementa el riesgo de infección en los animales vulnerables y la prevalencia parasitaria $(13,16)$.

En diferentes regiones del mundo se han identificado las siguientes especies y frecuencias parasitarias en gatos: Ancylostoma braziliensis, $25 \%$ (10); Ancylostoma tubaeforme, entre 14,7 y $44 \%(6,10,17) ;$ T. cati, del 0,8 al $79 \%(6,16,18,19)$, y Dipylidium caninum, entre 14 y $47 \%(9-11,18)$. Las prevalencias de las especies halladas en el presente estudio se encuentran dentro de los rangos reportados a nivel mundial.

Cuando se consideró la variable edad, en este estudio se encontró una mayor prevalencia de helmintos intestinales en los gatos mayores de 4 años con la técnica de Kato-Katz y en los animales con edades entre 1 y 4 años mediante la técnica de Ritchie. Curiosamente, los animales menores de un año de edad presentaron la menor prevalencia con ambas técnicas, pues normalmente se esperaría una mayor prevalencia de parasitismo en este grupo, dado que la inmunidad específica contra helmintos a esta edad no se ha desarrollado completamente. Por ejemplo, para T. caticomienza a manifestarse a partir de la quinta semana de edad (20); además, los cachorros se pueden infectar por vías de transmisión transplacentaria y transmamaria, por lo que el animal puede adquirir la parasitosis antes de nacer o desde el mismo momento en que empieza a alimentarse de la madre.

Los valores presentados en este trabajo no coinciden con algunos reportes, en los que hallaron que los helmintos intestinales son más comunes en gatos jóvenes que en gatos adultos (21), ya que la principal vía de transmisión en los gatos jóvenes es la transmamaria, a diferencia de los adultos que adquieren la infección por consumo de huéspedes paraténicos (22). Fromont, et al., reportaron mayor frecuencia de parásitos intestinales en felinos mayores de un año (19). Sin embargo, Overgaauw reportó mayores prevalencias en gatos mayores de seis meses (23); Luty, et al., encontraron frecuencias más elevadas $(32,5 \%)$ de $T$. cati en animales mayores de tres meses de edad (24); estos hallazgos no coinciden con la alta frecuencia de $T$. cati en gatos mayores de un año de edad encontrada en el presente estudio.

La condición corporal de la mayoría de los felinos evaluados fue buena y este fue el grupo de animales con menor frecuencia de helmintos 
intestinales. Si bien la presencia de parásitos se correlaciona negativamente con la condición corporal del gato, esto se puede explicar porque la respuesta inmunitaria del animal puede de alguna forma controlar la infección (21).

En el presente estudio, la frecuencia de hembras parasitadas fue ligeramente superior a la de los machos analizados, pero estas diferencias no fueron estadísticamente significativas. Estos resultados son semejantes a lo reportado por Mohammad, et al., quienes tampoco encontraron una diferencia significativa en las prevalencias de parasitosis intestinales estratificadas por sexo de los animales investigados, por lo que consideran que el sexo no es un factor determinante para la infección con estos agentes patógenos $(6,25)$.

Toxocara cati fue el parásito que se observó con mayor frecuencia en ambas técnicas en el presente estudio, seguido por parásitos del género Ancylostoma, lo cual difiere con lo reportado por Baker, et al., quienes encontraron una mayor frecuencia de este parásito en gatos adultos (10). En este estudio se evidenció una mayor frecuencia en gatos menores de un año con ambas técnicas de diagnóstico.

Hayat, et al., encontraron $77 \%$ de parasitosis única, el $15 \%$ de los gatos estaban parasitados con dos especies y $7 \%$ con tres especies (26), y Saito, et al., reportaron que el $61 \%$ de los gatos estudiados presentaban parasitosis única y $35 \%$ de ellos tuvieron dos especies; estos autores no refieren datos para más de tres especies. Las tendencias de las parasitosis únicas, de dos y hasta de tres especies, coinciden con lo hallado en este trabajo.

Según Tizard, las infecciones parasitarias presentan un comportamiento tal que la menor cantidad de individuos alberga a una gran cantidad de los parásitos (27). Esto podría explicar la mayor frecuencia de las parasitosis únicas. Este autor señala que la competencia que se puede generar por el hábitat y los nutrientes interviene en el progreso de las infecciones causadas por los helmintos. Por lo tanto, se esperaría un control del número y la composición de las poblaciones parasitarias, ya que se espera una menor competencia cuando existe un menor número de especies parasitarias $y$, por consiguiente, una gran disponibilidad de los recursos alimenticios, seguido de un aumento de la supervivencia parasitaria (19).

En conclusión, se observó una elevada prevalencia de parasitosis intestinales en los gatos estudiados en el departamento del Quindío; el 41,32 \% y el $46,28 \%$ resultaron parasitados con alguna especie de helminto adulto, según la presencia de huevos en las heces de los animales procesados mediante las técnicas de concentración de Ritchie y de KatoKatz, respectivamente.

\section{Agradecimientos}

Al Centro de Investigaciones Biomédicas de la Universidad del Quindío, al Instituto Seccional de Salud del Quindío y a la Secretaría de Salud de Armenia.

\section{Conflicto de interés}

Los autores declaramos que no existen conflictos de interés con la institución donde laboramos, ni existen relaciones financieras ni personales con otras personas u organizaciones que puedan influir o sesgar la forma de presentar los resultados.

\section{Financiación}

El presente trabajo se hizo con recursos propios del Grupo de Inmunología Molecular, adscrito a la Facultad de Ciencias de la Salud de la Universidad del Quindío.

\section{Referencias}

1. Robertson ID, Irwin PJ. Lymbery AJ, Thompson RC. The role of companion animals in the emergence of parasitc zoonoses. Int J Parasitol. 2000;30:1369-77. http://dx.doi. org/10.1016/S0020-7519(00)00134-X

2. Chandler EA, Gaskell CJ, Hilbery DR. Medicina y terapéutica felina. 1aㅡ edición. Zaragoza: Acribia; 1990. p. 415-24.

3. Chin J. El control de las enfermedades transmisibles. 17 edición. Washington, D.C.: Organización Panamericana de la Salud; 2001. p. 748.

4. Hendrix C, Bruce H, Kellman N, Harrelson G, Bruhn B. Cutaneous larva migrans and enteric hookworm infections. J Am Vet Med Assoc. 1996;209:1763-7.

5. Fisher M. Toxocara cati: An understimated zoonotic agent. Trends Parasitol. 2003;19:167-70. http://dx.doi.org/10.1016/ S1471-4922(03)00027-8

6. Calvete C, Lucientes J, Castillo J, Estrada R, Gracia $\mathbf{M}$, Peribáñez $\mathbf{M}$, et al. Gastrointestinal helminths parasites in stray cats from the mid-Ebro Valley, Spain. Vet Parasitol. 1998;75:235-40. http://dx.doi.org/10.1016/S03044017(97)00182-9

7. Hendrix C. Helminthic infections of the feline small and large intestines: Diagnosis and treatment. Vet Med. 1995;90:456-72.

8. Thrusfield M. Epidemiología veterinaria. Zaragoza: Editorial Acribia; 1990. p. 42.

9. Persson L. Gastro-intestinal parasites of cats. XIV Nordiske Veterinaer Kongres, Kobenhavn. Copenhagen: Foredragograpporter; 1982. p. 144-5. 
10. Baker MK, Lange L, Verster A, van der Plaat S. A survey of helminths in domestic cats in the Pretoria area of Transvaal, Republic of South Africa. Part 1: The prevalence and comparison of burdens of helminths in adult and juvenile cats. J S Afr Vet Assoc. 1989;60:139-42.

11. Fei-Chang $\mathbf{Y}$, Mo-Kang $\mathbf{M}$, Fei A, Mo K. Survey for endoparasitic zoonoses in stray dogs and cats in Taipei city. J Chin Soc Vet Sci. 1997;23:26-33.

12. Islam S, Deka D, Neog R, Baruah N, Das M, Borkakoty MR, et al. A note on the occurrence of certain helminth parasites in domestic cats (Felis catus domesticus) in Khanapara area of Guwahati, Assam. J Vet Parasitol. 1999;13:161-2.

13. Cordero del Campillo M, Rojo V, Martínez F, Sánchez A, Hernández R, Navarrete L, et al. Parasitología Veterinaria. Madrid: McGraw-Hill; 2000. p. 3-21.

14. Giraldo MI, García NL, Castaño JC, Gómez JE, Bedoya AM, Lora FM. Resultados preliminares sobre prevalencia de parásitos intestinales en perros y gatos del departamento del Quindío. Seinvestiga. Revista Médica Estudiantil de la Universidad del Quindío. 2003;7:7.

15. Acha P, Syfres B. Zoonosis y enfermedades transmisibles comunes al hombre y a los animales. $2^{\mathrm{a}}$ edición. Washington, D. C.: Organización Panamericana de la Salud/Organización Mundial de la Salud; 1986. p. 727-864.

16. Botero D, Restrepo M. Parasitosis humanas. Quinta edición. Medellín: Corporación para Investigaciones Biológicas; 2012. p. 511-30.

17. Schuster R, Kaufmann A, Hering S, Schuster R. Investigations on the endoparasite fauna of the domestic cat in eastern Brandenburg. Berl Munch Tierarztl Wochenschr. 1997;110:48-50.
18. Engbaek K, Madsen H, Olesen L, Larsen S. A survey of helminthes in stray cats from Copenhagen with ecological aspects. Zeitschrift fur Parasitenkunde. 1984;70:87-94.

19. Fromont E, Morvilliers L, Artois M, Pontier D. Parasite richness and abundance in insular and mainland feral cats: Insularity or density. Parasitology. 2001;123:143-51. http:// dx.doi.org/10.1017/S0031182001008277

20. Alarcón V, Bautista J. Efectividad del febendazol en suspensión al $10 \%$ en helmintiasis caninas en Bogotá (tesis). Santa $\mathrm{Fe}$ de Bogotá: Universidad de Ciencias Aplicadas y Ambientales; 1989.

21. Lightner L, Chistensen BM, Beran GW. Epidemiologic findings on canine and feline intestinal nematode infections from records of the lowa State University Veterinary Clinic. J Am Vet Med Assoc. 1978;172:564-7.

22. Martin WS, Meek HA, Willerberg P. Veterinary epidemiology. Principles and methods. Ames: lowa State University Press; 1987. p. 343.

23. Overgaauw PA. Toxocara infections in dogs and cats and public health implications. Vet Q. 1998;20(Suppl.1):S97-8.

24. Luty T, Mizgajska H. Prevalence of Toxocara spp. and other intestinal parasites in dogs and cats. Medycyna Weterynaryjna. 1999;11:759-61.

25. Mohammad Z, Seyed MS, Bahador S. Prevalence of Toxocara cati and other intestinal helminthes in stray cats in Shiraz. Trop Biomed. 2007;24:39-43.

26. Hayat C, Maqbool A, Hayat B, Badar N, Alam M. Prevalence and chemotherapy of intestinal parasites of domestic cats in Faisalabad, Pakistan. Iraqi J Vet Sci. 1999;12:109-13.

27. Tizard RI. Inmunología Veterinaria. 5a edición. México, D. F.: McGraw-Hill Interamericana; 1996. p. 567. 